\title{
Hypothalamic lesions and food/water intake in the Barbary dove'
}

\section{PETER WRIGHT. ${ }^{2}$ Institute of Experimental Psichology, Oxford, England}

Body'weight, food and water intake, and rectal temperature, in the Barbary dove, were measured daily during ad libitum food and water intake, both before and after placing radiofrequency lesions in the hypothalamus. One group of lesions resulted in a short-term aphagia in the birds, during which they showed an inability to mandibulate. Another lesion group produced a much longer aphagia in which the animals appeared to have a motirational deficiency.

Extensive information is available on the effects of hypothalamic lesions on the food and water intake of mammals (Anand, 1961) yet little attention has so far been devoted to the avian brain. During the course of an experiment in which discrete bilateral lesions have been placed systematically throughout the hypothalamus of the Barbary dove (Streptopelia risoria), two distinct kinds of feeding disturbance have been found. One is a short-term effect, in which the animals appear highly motivated to eat but are unable to perform the consummatory act of eating; the other is a long-term effect in which the animals display no interest in food. Feldman et al (1957) have previously reported aphagia in chickens resulting from destruction of scattered sites in the anterior, mid and posterior hypothalamus. These animals showed almost no voluntary activity and some had associated disturbances in temperature regulation. A permanent adipsia, with no accompanying aphagia, has also been reported in the chicken by Lepkovsky \& Yasuda (1967) following electrolytic lesions in the anterior hypothalamus.

\section{METHODS}

The Ss in the present report were adult Barbary doves (Streptopelia risoria) bred in the Institute colony. They were maintained on an artificial day length ( $8 \mathrm{~h}$ light, $16 \mathrm{~h}$ dark) in a temperature controlled room (25-30 deg C), visually isolated from each other in individual cages, with continuous access to food and water. Daily measurements of food and water intake, bodyweight and rectal temperature were recorded for a period of two weeks before and a minimum of three weeks after lesion. Radio frequency lesions were done with a Grass LM3 lesion maker via electrodes made from $27 \mathrm{G}$ stainless steel hypodermic tubing insulated to within $0.2 \mathrm{~mm}$ of the tip. During this procedure the birds were anesthetized with Equithesin and held in a modified Trent-Wells stereotaxic instrument. At the termination of the experiment, the birds were sacrificed and the brains perfused with $10 \%$ formol alcohol. Serial sections were cut at $15 \mu$ intervals in the plane of the electrode insertion and every fifth section was stained with a modified Weil's technique (those in the neighborhood of the lesion alternately with Glees and Nissl stains).

\section{RESULTS AND DISCUSSION}

In $10 \mathrm{Ss}$, a period of complete aphagia lasting from 3-7 days was observed. These animals appeared highly motivated to eat, engaging in continuous bill sweeping movements and pecking into bowls of wheat and millet seeds, but were unable to pick up a seed and move it to the back of the mouth prior to swallowing. When the birds had been aphagic for three days, they were force-fed by having seeds dropped at the back of their throats. This procedure demonstrated that swallowing was completely intact, and that the feeding deficit was one of mandibulation alone. Accuracy in pecking at seed on the ground was not impaired, and other activities involving beak movements such as preening were unaffected. There were no disturbances in temperature regulation other than the normal drop which McFarland \& Wright (1968) show in intact Barbary doves under conditions of food deprivation. Recovery from aphagia was complete in all but one $S$, which began to eat a small grain seed (millet) after seven days, but never regained the ability to eat adequate amounts of a large grain seed (wheat). Initially, the aphagia was accompanied in every case by adipsia. Animals that showed no spontaneous drinking could drink water if held with the bill immersed such that the mouth made contact with the water surface, again demonstrating intact swallowing. The recovery from adipsia always preceded recovery from aphagia (mean recovery periods for the short-term animals were: aphagia, 4.8 days: adipsia, 2.7 days); this finding is in marked contrast to the rat (Teitelbaum, 1954; Morrison, 1958; Epstein, 1964).

In five other Ss, a longer deficit occurred. Two birds recovered after 17 and 20 days, respectively, another was still aphagic at 35 days when it was killed. All three were initially adipsic for two days. The two other birds were aphagic and adipsic for a 12-day period, but this was accompanied by a drastically lowered body temperature necessitating their placement in a climatically-controlled cabinet. They were hydrated by tube and force-fed during this period and then sacrificed. No responses to food were shown by any of these during the period of aphagia. Lesions in other regions of the hypothalamus did not result in deficits of feeding and drinking and will be reported elsewhere $(\mathrm{N}=20$; aphagia, 0.5 days: adipsia, 0.1 days).

Histology (see Fig. 1) showed for the short-term animals, lesions rostrol to the anterior commissure, ? $\mathrm{mm}$ from the midline, and disrupting the lateral extremeties of the lateral forebrain bundle. Animals which exhibited long-term aphagia without accompanying hypothermia. had lesions at the same level, but disrupting the medial portions of the lateral forebrain bundle. The remaining two hypothermic birds (not illustrated) showed extensive destruction of the medial hypothalamus at this level. The avian lateral forebrain hundle has a great increase in the number of fibres and is more richly

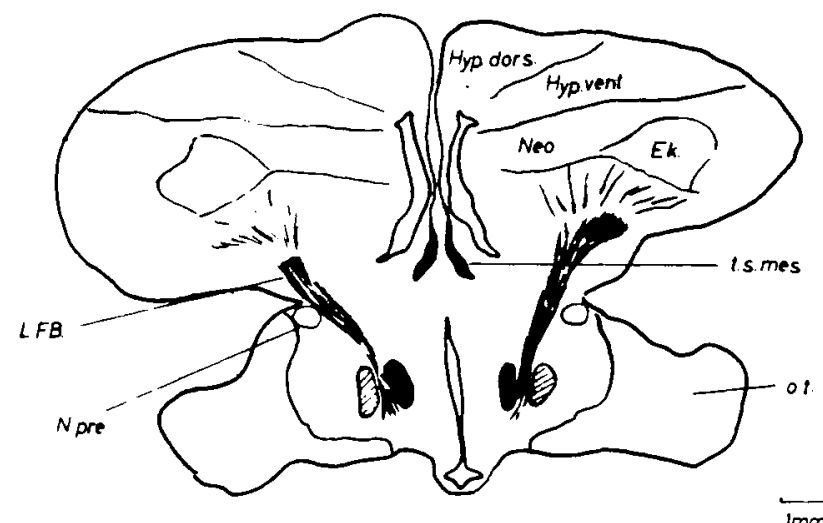

$1 \mathrm{~mm}$

Fig. 1. A frontal section of the barbary dove's brain showing the loci of the lesions: Ek., Ektostriatum; Hyp. dors., Hyperstriatum dorsalis; Hyp. vent., Hyperstriatum ventralis; Neo., Neostriatum: L.F.B., Lateral Forebrain Bundle: N. pre., Nucleus pretectalis; O.t. Optic tract; t.s.mes., tractus septo-mesencephalicus; lesions producing long-term aphagia; $2 Z$ lesions producing short-term aphagia. 
distributed than its homologue in reptiles, and includes connections between the striatal areas and the diencephalon. Harwood \& Vowles (1966) have shown that electrical stimulation of the neostriatum and paleostriatum in Barbary doves facilitates feeding behaviour. Akerman (1960) obtained feeding and pecking in pigeons from stimulation of sites in the lateral hypothalamus somewhat anterior to the anterior commissure and medial to the lateral forebrain bundle in the basal centres of the ventro-medial forebrain wall. This corresponds well with the region reported here, destruction of which results in aphagia. It also appears that both motivational and sensory-motor disturbances may result from destruction of two closely adjacent regions in the avian hypothalamus. Morgane (1961) has produced evidence for distinct "feeding" and "hunger-motivating" systems in the lateral hypothalamus of the rat, and the question as to whether aphagia is due to a motor failure or a motivational deficit has been raised by Baillie \& Morrison (1963) and by Rogers, Epstein, \& Teitelbaum (1965). Experiments are currently in progress in this laboratory to further clarify these functional differences in aphagia using an operant conditioning technique.

$$
\text { REFERENCES }
$$

AKERMAN, B., ANDERSSON, B., FABRICIUS, E., \& SVENSSON, L. Observations on central regulation of body temperature and of food and water intake in the pigeon (Columba livia). Acta Physiologica Scandinavica, 1960, 50, 328-336.

ANAND, B. K. Nervous regulation of food intake. Physiological Review, $1961,41,677-708$.
BAILLIE, P., \& MORRISON, S. D. The nature of the suppression of food intake by lateral hypothalamic lesion in rats. Journal of Physiology, 1963, 165, 227-245.

FELDMAN, S. E., LARSSON, S., DIMICK, M. K., \& LEPKOVSKY, S. Aphagia in chickens. American Journal of Physiology, 1957, 191, 259-261.

EPSTEIN, A. N., \& TEITELBAUM, P. Severe and persistent deficits in thirst produced by lateral hypothalamic damage. In M. J. Wayner (Ed.), Thirst. New York: Pergamon, 1964.

HARWOOD, D., \& VOWLES, D. M. Forebrain stimulation and feeding behaviour in the ring dove (Streptopelia risoria). Journal of Comparative \& Physiological Psychology, 1966, 62, 388-396.

LEPKOVSKY, S., \& YASUDA, M. Adipsia in chickens. Physiology \& Behavior, 1967, 2, 45-47.

McFARLAND, D. J., \& WRIGHT, P. Water conservation by inhibition of food intake. Physiology \& Behavior, in press.

MORGANE, P. J. Distinct "feeding" and "hunger motivating" systems in the lateral hypothalamus of the rat. Science, 1961, 133,887.

MORRISON, S. D., BARNETT, R, J, \& MAYER, J. Localization of lesions in the lateral hypothalamus of rats with induced aphagia and adipsia. American Journal of Physiology, 1958, 193, 230-234.

RODGERS, W. L., EPSTEIN, A. N., \& TEITELBAUM, P. Lateral hypothalamic aphagia: Motor failure or motivational deficit? American Journal of Physiology, 1965, 208(2), 334-342.

\section{NOTES}

1. This research was supported by a grant from the Science Research Council. The author held a scholarship from the Medical Research Council and is grateful for this assistance.

2. Now at the Department of Psychology, University of Edinburgh. 